EXTENDED REPORT

\title{
Lens fluorescence and metabolic control in type 1 diabetic patients: a 14 year follow up study
}

\author{
L Kessel, B Sander, P Dalgaard, M Larsen
}

Br J Ophthalmol 2004;88:1169-1172. doi: 10.1136/bjo.2003.028779

Background/aims: In this prospective study the authors followed the post-translational modification of the proteins of the lens of the eye by fluorometry over a period of 14 years in patients with type 1 diabetes mellitus.

Methods: Twenty patients were examined at 6 years (baseline) and 20 years (follow up) after onset of

See end of article for authors' affiliations

.....................

Correspondence to: L Kessel, Department of Ophthalmology, Herlev Hospital, Herlev Ringvei 75, DK-2730 Herlev, Denmark;

line.kessel@dadlnet.dk

Accepted 1 February 2004 diabetes mellitus. Glycaemic levels were assessed from $\mathrm{HbAlc}$ measured at regular intervals from the onset of diabetes mellitus.

Results: Lens fluorescence at follow up was significantly related to mean $\mathrm{HbAlc}$ during the study period and to lens fluorescence at baseline $(p<0.0001)$. Sixty per cent of the variation in rate of increase in lens fluorescence during the study period was statistically attributable to glycaemia levels.

Conclusion: The results confirm that the rate of fluorophore accumulation in the lens of adult diabetic patients is increased in proportion to glycaemic control. This parameter alone is, however, not sufficient to explain the entire variation in lens fluorophore accumulation. Consequently, lens fluorescence must be influenced by other factors before initiation and during the study period, and possibly even before onset of diabetes mellitus. The underlying mechanism could be a variation in susceptibility to lens protein denaturation by glycation.
L

ens fluorescence (LF) increases cumulatively throughout

life at a rate that is strongly influenced by blood glucose

- levels in both healthy and diabetic ranges of glucose metabolism. ${ }^{1-3}$ It is caused by irreversible protein denaturation due to formation of advanced glycation end products. ${ }^{4}$ The process of advanced glycation end product formation takes place in all tissues ${ }^{5}$ and is of importance for the pathogenesis of diabetic long term complications. ${ }^{6}$ Indeed, LF is increased in patients with diabetic long term complications compared with diabetic patients without complications. ${ }^{7}$ The formation of advanced glycation end products on lens proteins in situ is irreversible and because there is no turnover of lens proteins, the intensity of fluorescence of the lens may be regarded as a summary measure of the amount of sugar induced protein denaturation that has occured during the entire life span of an individual.

Cross sectional studies suggest that lens fluorometry offers an opportunity to assess a life long glycaemic index. ${ }^{89}$ Previously, a follow up study on LF in healthy subjects was presented $^{10}$ but no long term studies of LF in relation to glucose metabolism have been reported. The aim of the present prospective study was to follow LF in relation to the degree of hyperglycaemia.

\section{MATERIALS AND METHODS}

All patients examined in our study had type I diabetes mellitus as evidenced by insulin therapy since onset and low $\mathrm{C}$ peptide shortly after onset of the disease. The present study is the 14 year follow up of a previous cross sectional study conducted 6 years after the onset of diabetes (baseline examination). ${ }^{8}$ Here, we report the status at 14 years after baseline-that is, 20 years after onset of diabetes mellitus.

Patients were initially recruited from a database of all diabetic patients attending the ophthalmic service at the Steno Diabetes Center, Gentofte, Denmark. Patients were polled to represent two extremes of metabolic control on basis of their mean $\mathrm{HbAlc}$ between onset of diabetes mellitus and the baseline examination. One group of patients had been in good metabolic control (mean HbAlc 6.5\% from onset to baseline examination 6 years after onset) and the other group had been in poor metabolic control (mean HbAlc $10.1 \%) .{ }^{8}$ Thirty six patients were enrolled at baseline. At follow up, 14 years after the baseline examination, two patients had died, three had emigrated or moved, and two had undergone bilateral cataract surgery, thus leaving 29 patients eligible for inclusion. The 20 patients who volunteered to participate had attended regular diabetes care at specialised diabetes clinics in the interval between the two examinations. All patients gave their written informed consent to participate in the study after the nature and possible consequences of the study had been carefully explained. The study was approved by the regional medical ethics committee and followed the tenets of the Declaration of Helsinki.

Complication status and HbAlc data from the study period were obtained from the patients' medical records. Long term complications were defined as microalbuminuria (30$300 \mathrm{mg} / 24 \mathrm{~h}$ ), macroalbuminuria (>300 mg/24 h), arterial hypertension (>140/90 $\mathrm{mm} \mathrm{Hg}$ ) or ongoing pharmaceutical antihypertensive treatment, or sight threatening retinopathy requiring photocoagulation treatment (macular oedema and/or proliferative retinopathy). Regular measurements of HbAlc (one to four times annually) were available for 18 of the 20 patients from baseline examination to follow up. For the remaining two patients complete HbAlc datasets were not available.

At follow up, all patients were examined by ocular biomicroscopy in full mydriasis. Best corrected visual acuity was determined using a Snellen chart. Retinopathy was graded according to a modified Eurodiab protocol on the basis of two field 60 degree stereoscopic fundus photographs, one pair centred at the fovea and one pair centred on the optic nerve head. ${ }^{11}$ Tobacco consumption was assessed as

Abbreviation: LF, lens fluorescence. 
pack years ever smoked, one pack year equalling 20 cigarettes per day for a period of one year.

Lens fluorescence was measured on the undilated eye using a non-invasive ocular fluorometer (Fluorotron, OcuMetrics, Mountain View, CA, USA). The same instrument was used at baseline and follow up. Excitation was at 430 $490 \mathrm{~nm}$, and detection was at 530-630 nm. Fluorescence was measured in intraocular intervals of $0.125 \mathrm{~mm}$, the measurements being restricted to the cornea, anterior chamber, and the lens. Three scans were performed per eye and LF was defined as the mean value of the five highest readings of the anterior lens peak on each scan. Lens fluorescence was averaged over three scans from each eye and the mean value of both eyes was used for statistical analyses. The same procedure was applied for corneal fluorescence. Fluorescence measurements were calibrated to an external fluorescein standard and are reported in ng equivalent fluorescein concentration (ng $\mathrm{f}-\mathrm{eq} / \mathrm{ml}$ ). A drift in calibration between baseline and follow up was compensated for by age corrected normalisation to data from more than 50 healthy subjects examined concurrently with the two visits. The calibration factor was 0.23 which was added to the $\log 10$ values of LF at baseline examination.

\section{Statistical procedures}

Lens fluorescence values were transformed into logarithmic values $(\log 10)$ to obtain a normal distribution with constant variance. Fluorescence data presented have been backtransformed to a geometrical mean with $95 \%$ confidence intervals. The rate of annual increase in LF was determined as the difference in LF between baseline and follow up examination divided by time interval and is expressed as ng $\mathrm{f}$-eq/ml/year. Significance testing of differences was performed using Student's $t$ test. The correlation between variables was calculated using Pearson's coefficient of correlation. Multiple linear regression analysis was used to test relations between variables. Statistical analyses were performed using the R software version 1.6.2 (http://www. r-project.org).

\section{RESULTS}

The 20 patients available for follow up were representative of the baseline study population of 36 subjects with respect to age, LF, and HbAlc. None of the examined patients had opacities of the refractive media within $2 \mathrm{~mm}$ of the optical axis of the eye that could potentially interfere with fluorescence measurements. The bimodal distribution of HbAlc-levels at baseline had largely been preserved at follow up for the population as well as for the individual patients, except for one patient who had suffered a prolonged period of poor metabolic control during a pregnancy (fig 1). This patient was a statistical outlier and has been omitted from the results presented below which are based on the remaining 19 patients. Clinical characteristics of the study population are given in table 1 .

\section{Lens fluorescence}

Lens fluorescence increased significantly from baseline to follow up examination $(\mathrm{p}<0.0001$; table 1$)$. Lens fluorescence (log 10 values) at follow up was significantly related to mean HbAlc in the 14 year long study period and to LF (log 10 values $)$ at baseline examination $(\mathrm{p}<0.0001$; fig 2 and equation 1 ):

$$
\begin{aligned}
\mathrm{LF}(\text { follow up })= & 0.74701 \mathrm{LF}(\text { baseline })+0.02488 \mathrm{HbAlc} \\
& +0.64814
\end{aligned}
$$

The mean (standard deviation) annual increase in LF between baseline and follow up 14 years later was 16.65

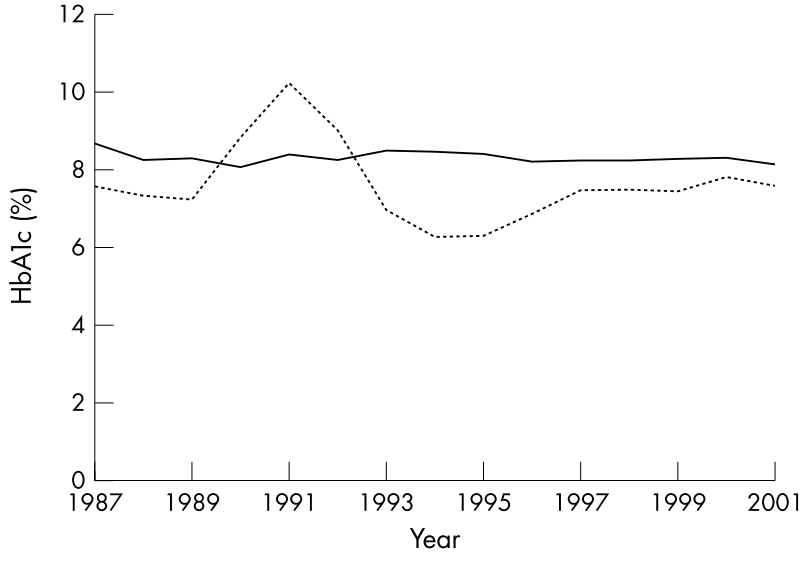

Figure $1 \mathrm{HbAlc}$ between baseline (in 1987) and follow up examination (in 2001). The bold line represent the mean of all patients $(n=19)$ except for one patient who had an uncharacteristic oscillation in metabolic control (thin line). This patient was excluded from the final statistical analyses.

Table 1 Clinical characteristics of the study population

\begin{tabular}{llll}
\hline & Baseline & Follow up & p Value \\
\hline Number of patients & 19 & 19 & \\
Age (years) & $30.0(8.8)$ & $44.0(8.8)$ & \\
$\begin{array}{l}\text { Duration of diabetes } \\
\text { (years) }\end{array}$ & $5.8(1.2)$ & $19.8(1.2)$ & \\
Mean HbAlc (\%)* & $8.0(1.9)$ & $7.8(2.2)$ & 0.71 \\
Lens fluorescence & 443.3 & 675.5 & $<0.0001$ \\
(ng f-eq/ml) & $(238.0$ to 825.8$)$ & $(404.4$ to 1128.3) & \\
\hline
\end{tabular}

Data are presented in mean (SD) except for fluorescence values which are given in mean ( $95 \%$ confidence interval) after backtransformation from logarithmic values.

*Mean $\mathrm{HbAlc}$ at baseline examination was calculated as the mean of all $\mathrm{HbAlc}$ values from onset of diabetes to baseline examination. Mean $\mathrm{HbAlc}$ at follow up examination was calculated at the mean of all $\mathrm{HbAlc}$ values from baseline to follow up examination. $p$ Values were calculated using a paired $t$ test.

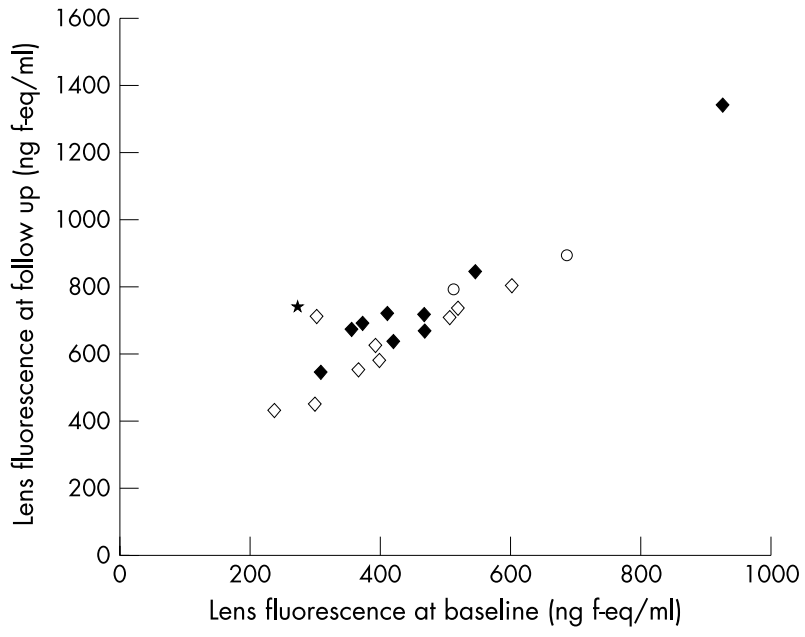

Figure 2 Lens fluorescence at baseline examination and follow up examination. The mean level of $\mathrm{HbAlc}$ from baseline to follow up 14 years later was $>8 \%$ in nine patients (solid symbols) and $\leqslant 8 \%$ in nine patients (open symbols). HbAlc from baseline to follow up was unavailable in two patients (circles). A single patient (marked *) was a statistical outlier and was excluded from the results presented. 


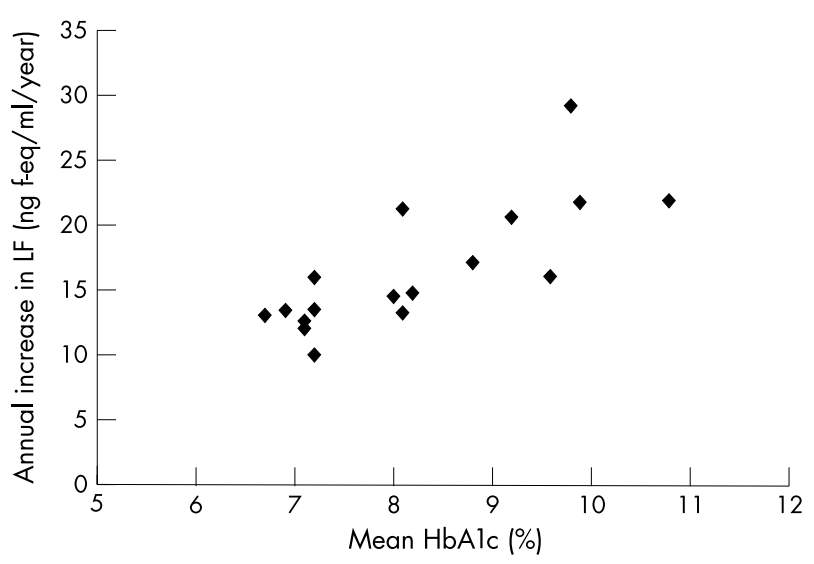

Figure 3 Annual increase in lens fluorescence (LF) from baseline to follow up in relation to mean $\mathrm{HbAlc}$ in the same period $(n=17)$. The two patients in whom mean $\mathrm{HbAlc}$ could not be assessed are omitted from the graph.

( $\mathrm{SD} 4.69$ ) $\mathrm{ng} \mathrm{f}-\mathrm{eq} / \mathrm{ml} / \mathrm{year}$. The rate of annual increase in LF was significantly related to the mean $\mathrm{HbAlc}$ in the period between baseline and follow up examination $(p=0.0003$; fig 3 and equation 2):

$$
\text { Annual increase }(\mathrm{LF})=3.04511 \mathrm{HbAlc}_{\text {mean }}-8.42855
$$

Thus, a patient with a mean $\mathrm{HbAlc}$ of $7 \%$ had a predicted rate of increase in LF of $12.89 \mathrm{ng} \mathrm{f-eq/ml} /$ year and a patient with a mean HbAlc of $8 \%$ had a predicted rate of increase in LF of $15.93 \mathrm{ng} \mathrm{f}-\mathrm{eq} / \mathrm{ml} / \mathrm{year}$, the rate of increase growing by approximately $20 \%$ when the glycated fraction of HbAlc was increased by 1 percentage point. The variations in HbAlc were found to account for $57.51 \%$ of the variation (adjusted $\mathrm{R}^{2}$ ) in the rate of increase in lens fluorescence. No significant effect of tobacco smoking on LF values was found in the present population $(\mathrm{p}=0.17)$.

\section{Diabetic long term complications}

A total of eight patients had developed long term complications of diabetes, all such events occurring between baseline ( six years after onset of diabetes mellitus) and follow up (20 years after onset). Complications included hypertension (7), microalbuminuria (3), and diabetic retinopathy treated by photocoagulation (2). None of the patients had developed neuropathy.

Patients with complications had had a higher mean HbAlc in the period from baseline to follow up than patients without complications $(\mathrm{p}=0.05)$, with respective mean (SD) HbAlc-levels of 8.9 (1.0) \% in patients with complications versus 7.7 (1.2) \% in patients without complications. Patients with complications had higher age corrected LF than patients without complications $(p=0.02)$. The effect disappeared after inclusion of cumulative HbAlc in the descriptive model $(p=0.17)$, presumably because LF and cumulative HbAlc are both measures of integrated glycaemia. Likewise, patients who eventually developed complications also had higher levels of LF values at baseline $(p=0.02)$ but this difference also disappeared after incorporating the effect of HbAlc from onset of diabetes to baseline $(p=0.34)$.

At follow up, five patients (25\%) had no diabetic retinopathy in either eye, three had developed mild retinopathy in one eye only, nine had mild or moderate retinopathy on both eyes, and two had received bilateral retinal photocoagulation treatment for macular oedema and proliferative retinopathy, respectively. The degree of retinopathy was found to increase with the mean (SD) HbAlc during the study period, from $7.2(0.5) \%$ in patients without retinopathy to $8.4(1.2) \%$ in patients with mild/moderate retinopathy and $9.8(0.2) \%$ in patients who had received retinal photocoagulation. There was no obvious evidence of a relation between LF and retinopathy with mean fluorescence levels of 556.72 (95\% CI 531.23 to 583.40 ) (ng f-eq/ml) in patients without retinopathy, 746.04 (95\% CI 466.52 to 1193.05 ) in patients with mild/moderate retinopathy, and 603.60 (95\% CI 429.09 to 849.08 ) in patients who had received retinal photocoagulation.

\section{DISCUSSION}

Type 1 diabetes is a chronic disease that requires intensive treatment and care from health professionals and the patient in order to avoid serious long term complications such as retinopathy and nephropathy. The risk of developing complications is closely related to the level of hyperglycaemia ${ }^{12}$ though metabolic control cannot fully account for the variation in frequency and severity of complications. ${ }^{13}$

One of the specific aims of this study was to examine the relation between LF and metabolic control prospectively in a group of type 1 diabetic patients in whom HbAlc had been measured regularly during the 14 year long study period. Our observations strongly support the theory that LF is related to the mean level of HbAlc over a period of more than a decade-the mean HbAlc accounting for nearly $60 \%$ of the variation in the rate of increase in lens fluorescence. We have also found that the likelihood of having diabetes complications increases with the fluorescence of the lens, but cumulative HbAlc remains the better predictor of the two.

A prominent finding of our study was that LF at follow up examination was very closely related to LF at baseline examination. The same observation was previously made in healthy subjects. ${ }^{10}$ Patients thus tend to keep a predetermined level of LF that was set already at baseline examination and possibly even before the onset of diabetes mellitus, a level which may only be partly sensitive to glycaemic control as assessed by HbAlc. This indicates that LF to some extent is determined by factors unrelated to glycaemia. This is likely to include genetic factors unrelated to diabetes mellitus, as previously shown. ${ }^{3}$ This study indicates that genetic factors could be of importance in diabetic patients as well. It has been shown that the severity of diabetic complications is partly genetically determined. ${ }^{14}{ }^{15}$ The common pathogenic factor between LF and diabetic complications is hyperglycaemia resulting in tissue damage by advanced glycation end products. Lens fluorescence measurements may thus be an indicator not only of glycaemic control but also of genetic susceptibility to tissue damage by glycation.

Other factors may need to be taken into account to explain the interindividual variation in lens fluorescence. In a previous study, we found that tobacco smoking affected LF to the same degree as glucose metabolism in healthy subjects ${ }^{3}$ probably due to its content of glycotoxins leading to formation of advanced glycation end products. ${ }^{16}{ }^{17}$ The finding was not repeated in the present study, most likely because blood glucose levels are so high in diabetic patients that it masks any effect caused by smoking.

In summary, our study suggests that in the absence of other information about prior levels of glycaemia, lens fluorometry is a useful marker of life long glycaemia in population studies where the interindividual variation in fluorescence attributable to factors other than glycaemia can be evenly distributed between groups. Furthermore, we have shown that the rate of increase in fluorophore accumulation is predetermined, possibly due to a genetically determined susceptibility to glycation which may also account for some 
of the interindividual variation in the complication rate of diabetes.

\section{ACKNOWLEDGEMENTS}

The study was presented in part at the 2002 annual EASDEC conference in Udine, Italy. The study received financial support from the Velux Foundation and through a Patient-Oriented Diabetes Research Career Award from the Juvenile Diabetes Research Foundation to Dr Larsen (grant number 8-2002-130).

\section{Authors' affiliations}

L Kessel, B Sander, M Larsen, Department of Ophthalmology, Herlev Hospital, University of Copenhagen, Denmark

P Dalgaard, Department of Biostatistics, University of Copenhagen, Denmark

\section{REFERENCES}

1 Lutze M, Bresnick GH. Lenses of diabetic patients "yellow" at an accelerated rate similar to older normals. Invest Ophthalmol Vis Sci 1991;32:194-9.

2 Siik S, Airaksinen PJ, Tuulonen A, et al. Lens autofluorescence in healthy individuals. Acta Ophthalmol (Copenh) 1991;69:187-92.

3 Kessel L, Hougaard JL, Sander B, et al. Lens ageing as an indicator of tissue damage associated with smoking and non-enzymatic glycation-a twin study. Diabetologia 2002;45:1457-62.

4 Monnier VM, Cerami A. Nonenzymatic browning in vivo: possible process for aging of long-lived proteins. Science 1981;211:491-3.

5 Ulrich P, Cerami A. Protein glycation, diabetes, and aging. Recent Prog Horm Res 2001;56:1-21.

6 Paul RG, Bailey AJ. Glycation of collagen: the basis of its central role in the late complications of ageing and diabetes. Int J Biochem Cell Biol 1996;28:1297-310.
7 Larsen M, Kier B, Bendtson I, et al. Lens fluorescence in relation to nephropathy in insulin-dependent diabetes mellitus. Graefes Arch Clin Exp Ophthalmol 1992;230:6-10.

8 Larsen M, Kjer B, Bendtson I, et al. Lens fluorescence in relation to metabolic control of insulin-dependent diabetes mellitus. Arch Ophthalmol 1989;107:59-62.

9 Koefoed TP, Hansen T, Larsen M, et al. Lens autofluorescence is increased in newly diagnosed patients with NIDDM. Diabetologia 1996:39:1524-7.

10 Van Best JA, Van Delft JL, Keunen JE. Long term follow-up of lenticular autofluorescence and transmittance in healthy volunteers. Exp Eye Res 1998:66:117-23

11 Aldington SJ, Kohner EM, Mever S, et al. Methodology for retinal photography and assessment of diabetic retinopathy: the EURODIAB IDDM complications study. Diabetologia 1995;38:437-44.

12 The effect of intensive treatment of diabetes on the development and progression of long-term complications in insulin-dependent diabetes mellitus. The Diabetes Control and Complications Trial Research Group. N Engl J Med 1993;329:977-86.

13 Zhang L, Krzentowski G, Albert A, et al. Risk of developing retinopathy in Diabetes Control and Complications Trial type 1 diabetic patients with good or poor metabolic control. Diabetes Care 2001;24:1275-9.

14 Clustering of long-term complications in families with diabetes in the diabetes control and complications trial. The Diabetes Control and Complications Trial Research Group. Diabetes 1997:46:1829-39.

15 Karamanos B, Porta $M$, Songini $M$, et al. Different risk factors of microangiopathy in patients with type I diabetes mellitus of short versus long duration. The EURODIAB IDDM Complications Study. Diabetologia 2000:43:348-55.

16 Cerami C, Founds $\mathrm{H}$, Nicholl I, et al. Tobacco smoke is a source of toxic reactive glycation products. Proc Natl Acad Sci USA 1997;94:13915-20.

17 Nicholl ID, Stitt AW, Moore JE, et al. Increased levels of advanced glycation endproducts in the lenses and blood vessels of cigarette smokers. Mol Med 1998;4:594-601 